\title{
Oscillation of a forced higher order equation
}

\author{
by Witold A. J. Kosmala (Boone, N.C.)
}

\begin{abstract}
We state and prove two oscillation results which deal with bounded solutions of a forced higher order differential equation. One proof involves the use of a nonlinear functional.
\end{abstract}

Introduction. The main objective of this paper is to present two oscillation results for bounded solutions of the differential equation

$$
x^{(n)}+p(t) x^{(n-1)}+q(t) x^{(n-2)}+H(t, x)=Q(t)
$$

where $n \geq 3$ is an integer and $H: \mathbb{R}^{+} \times \mathbb{R} \rightarrow \mathbb{R}$ is continuous, decreasing in its second variable and such that $u H(t, u)<0$ for all $u \neq 0$. Here $\mathbb{R}$ denotes the real line and $\mathbb{R}^{+}$the interval $[0, \infty)$. The differential equation $(*)$ has not been much studied under the assumptions on $H$ as described above. The only oscillation result known to the author is given in [5]. In that paper $Q(t)$ is identically zero and conditions on $H$ are stronger. There is no oscillation result known for $(*)$ with $H$ as described above in the case of $n$ even. As in [5], in this paper we also use a nonlinear functional to prove the result. This approach came in useful to Erbe [1], Heidel [2], Kartsatos [3], Kartsatos and Kosmala [4], and others in proving their theorems. In [6] the author also uses nonlinear functionals to prove a variety of asymptotic properties of the differential equation $(*)$. The reader might also wish to explore [7] where $H$ is different but some other assumptions as well as methods are similar.

In what follows, we say that $x(t), t \in\left[t_{x}, \infty\right) \subset \mathbb{R}^{+}$, is a solution of $(*)$ if it is $n$ times continuously differentiable and satisfies $(*)$ on $\left[t_{x}, \infty\right)$. The number $t_{x} \geq 0$ depends on the particular solution $x(t)$ under consideration. We say that the function is oscillatory if it has an unbounded set of zeros. Moreover, we say that a property $\mathrm{P}$ holds eventually or for all large $t$ if there

1991 Mathematics Subject Classification: Primary 34C10.

Key words and phrases: oscillation, nonlinear higher order equation, nonlinear functional.

Paper completed during author's sabbatical at the University of Alberta in Edmonton, Alberta, Canada. 
exists $T \geq 0$ such that $\mathrm{P}$ holds for all $t \geq T$. We denote by $C^{n}(I)$ the space of all $n$ times continuously differentiable functions $f: I \rightarrow \mathbb{R}$. We write $C(I)$ instead of $C^{0}(I)$. Throughout this paper we assume that $p \in C^{1}\left[t_{0}, \infty\right)$ and $q \in C\left[t_{0}, \infty\right)$ with

$$
2 q(t) \leq p^{\prime}(t)
$$

for $t \geq t_{0}$. Moreover, we assume that $S$ is a solution of

$$
S^{(n)}+p(t) S^{(n-1)}+q(t) S^{(n-2)}=Q(t)
$$

which tends to zero.

Lemma 1 in [5] can be extended to the forced equation without too much difficulty. For the sake of completeness we state it formally and provide the proof.

LEMMA. If $x$ is an eventually positive solution of $(*)$, then either $[x(t)-$ $S(t)]^{(n-2)} \leq 0$ or $[x(t)-S(t)]^{(n-2)}>0$ for all large $t$.

Proof. Suppose $x(t)>0$ and $2 q(t) \leq p^{\prime}(t)$ for all $t \geq t_{0} \geq 0$. Let $u=x-S$ with $t \geq t_{0}$. Then the equation $(*)$ becomes

(2) $\quad u^{(n)}(t)+p(t) u^{(n-1)}(t)+q(t) u^{(n-2)}(t)+H(t, u(t)+S(t))=0$.

Now, we suppose to the contrary that $u^{(n-2)}\left(t_{1}\right)=u^{(n-2)}\left(t_{2}\right)=0$ with $u^{(n-2)}(t)>0$ for $t_{0} \leq t_{1}<t<t_{2}$. This implies that $u^{(n-1)}(t) \not \equiv 0$ on $\left(t_{1}, t_{2}\right)$. Now, multiply $(2)$ by $u^{(n-2)}(t)$ and integrate from $t_{1}$ to $t_{2}$ to obtain

$$
\begin{aligned}
& \int_{t_{1}}^{t_{2}} u^{(n-2)}(t) H(t, u(t)+S(t)) d t \\
& \quad=\int_{t_{1}}^{t_{2}}\left(u^{(n-1)}(t)\right)^{2} d t-\int_{t_{1}}^{t_{2}}\left(q(t)-\frac{p^{\prime}(t)}{2}\right)\left(u^{(n-2)}(t)\right)^{2} d t>0 .
\end{aligned}
$$

Since the left hand side cannot be positive, we obtain a contradiction. Hence, the proof is complete.

This Lemma can be rephrased for an eventually negative solution as well.

THEOREM 1. Consider the differential equation $(*)$ with the following additional assumptions:

(i) $n \geq 3$ is an odd integer,

(ii) $p(t) \leq 0, q(t) \geq 0$ and

$$
t\left[q(t)-p^{\prime}(t)\right] \leq 2 p(t)
$$

eventually, and 
(iii) for any positive real constant $k$,

$$
-\int^{\infty} t^{2} H(t, \pm k) d t= \pm \infty
$$

Then every bounded solution of $(*)$ is oscillatory or tending to zero.

Remarks. (a) If $Q(t) \equiv 0$, then every bounded solution of (*) must oscillate.

(b) The function $p$ cannot be a negative constant because if it is, by assumption (ii) and (1), $q(t) \equiv 0$. But this contradicts condition (3).

(c) Suppose $p(t) \leq 0$ and $q(t) \geq 0$ eventually. Then assumption (1) does not imply assumption (3). Indeed, $p(t)=-1 / t$ and $q(t)=1 /\left(5 t^{2}\right)$ satisfy (1) but not (3). Moreover, condition (3) does not imply condition (1). For example, $p(t)=-1 / t^{5}$ and $q(t)=2.8 / t^{6}$ satisfy (3) but not (1). It can be proven, however, that if $p(t)$ satisfies

$$
p(t) \leq\left(\frac{t^{*}}{t}\right)^{4} p\left(t^{*}\right)
$$

with $t \geq t^{*}$ for any fixed $t^{*}>0$ for which $p\left(t^{*}\right)<0$ then, together with assumption (3), the condition (1) must hold.

(d) A familiar differential equation

$$
x^{\prime \prime \prime}-8 x=0
$$

fits all the assumptions of Theorem 1 . It is easy to verify that since three linearly independent solutions are $e^{2 t}, e^{-t} \sin \sqrt{3} t, e^{-t} \cos \sqrt{3} t$, all the solutions of this equation are either unbounded or bounded and oscillatory.

(e) Every homogeneous differential equation has a trivial bounded oscillatory solution. In particular, the differential equation

$$
x^{\prime \prime \prime}-\frac{1}{t^{4}} x^{\prime \prime}+\frac{1}{t^{6}} x^{\prime}-\left(1-\frac{1}{t^{4}}+\frac{1}{t^{6}}\right) x=0
$$

has a bounded oscillatory solution $x(t)=0$ and an unbounded solution $x(t)=e^{t}$ for $t>0$. Since the coefficient functions satisfy all the conditions in Theorem 1 , every solution of this equation is unbounded and/or oscillatory.

(f) We observe that the differential equation

$$
x^{\prime \prime \prime}-\frac{1}{t^{4}} x^{\prime \prime}+\frac{1}{t^{6}} x^{\prime}-\frac{1}{t} \arctan (t x)=-\frac{1}{t^{8}}\left(6 t^{4}+2 t+1\right)
$$

involves functions which satisfy all the required conditions in Theorem 1 , and hence, every solution of this equation is either unbounded, oscillatory or tending to zero. In fact, $S(t)=1 / t$ in the above equation.

(g) The differential equation $(*)$ has some applications in stock market fluctuations, generalized mechanics, and astrophysics. 
Proof of Theorem 1. We proceed by contradiction. Without loss of generality, we will assume that $x$ is a bounded, positive solution of $(*)$ which does not tend to zero, and we will also assume that all the conditions on the functions $p$ and $q$ are satisfied for $t \geq t_{0} \geq 0$. We let $u=x-S$ with $t \geq t_{0}$. Then equation $(*)$ can be written as equation (2). Also, by the above Lemma, we have either $u^{(n-2)}(t) \leq 0$ or $u^{(n-2)}(t)>0$. In order to prove the theorem, we need to consider both cases and find a contradiction in each.

Case 1 . We assume that $u^{(n-2)}(t) \leq 0$ for $t \geq t_{1} \geq t_{0}$. Moreover, we suppose that there exists $t_{2} \geq t_{1}$ such that $u^{(n-1)}\left(t_{2}\right)=0$. Then we get

$$
u^{(n)}\left(t_{2}\right)=-q\left(t_{2}\right) u^{(n-2)}\left(t_{2}\right)-H\left(t_{2}, u\left(t_{2}\right)+S\left(t_{2}\right)\right)>0 .
$$

Thus, $u^{(n-1)}(t)$ is increasing at any $t_{2}, t_{2} \geq t_{1}$, for which it is zero. Therefore, $u^{(n-1)}(t)$ cannot have any zeros larger than $t_{2}$. Moreover, $u^{(n-1)}(t)$ cannot be eventually negative, because together with the fact that $u^{(n-2)}(t) \leq 0$ we get $\lim _{t \rightarrow \infty} u(t)=-\infty$. Thus, $\lim _{t \rightarrow \infty}[x(t)-S(t)]=-\infty$. Since $\lim _{t \rightarrow \infty} S(t)=$ 0 , we have $\lim _{t \rightarrow \infty} x(t)=-\infty$, which contradicts the positivity of $x$.

We conclude that $u^{(n-1)}(t)>0$ eventually. However, this is also impossible because from $(2)$ we get $u^{(n)}(t)>0$ for all large $t$. Together with $u^{(n-1)}(t)>0$, this implies that $u^{(n-2)}(t)>0$ eventually. This again gives a contradiction. This takes us to the next case.

Case 2. We assume that $u^{(n-2)}(t)>0$ for $t \geq t_{3} \geq t_{0}$. Since $x(t)>$ 0 and $\lim _{t \rightarrow \infty} S(t)=0$, we have $u(t)=x(t)-S(t)>0$, which must be bounded (otherwise $x$ will be unbounded), which in turn implies that $u^{(n-3)}(t)<0$ for all $t \geq t_{4} \geq t_{3}$. Therefore, there exists $\varepsilon>0$ such that $u\left(t_{4}\right)=x\left(t_{4}\right)-S\left(t_{4}\right)>\varepsilon$ and $-\varepsilon<S(t)<\varepsilon$ for all $t \geq t_{4}$. Keeping in mind that $n$ is odd, we have $u^{\prime}(t)>0$ for $t \geq t_{5} \geq t_{4}$. This enables us to write

$$
u(t)+S(t)>u(t)-\varepsilon>u\left(t_{5}\right)-\varepsilon \equiv k>0 \quad \text { for all } t \geq t_{5} .
$$

We define the functional $G$ by

(4) $G(u(t))=2 u^{(n-3)}(t) u^{(n-1)}(t)+2 p(t) u^{(n-3)}(t) u^{(n-2)}(t)-\left[u^{(n-2)}(t)\right]^{2}$.

We will prove that $G(u(t))>0$ eventually by assuming to the contrary. So, let $t_{6} \geq t_{5}$ be such that $G\left(u\left(t_{6}\right)\right) \leq 0$. Note that if $t_{6}$ like this does not exist, we are done. So now, we write

$$
\begin{aligned}
G^{\prime}(u(t)) & \\
= & 2 u^{(n-3)}(t) u^{(n)}(t)+2 u^{(n-2)}(t) u^{(n-1)}(t)+2 p(t) u^{(n-3)}(t) u^{(n-1)}(t) \\
& +2 p(t)\left[u^{(n-2)}(t)\right]^{2}+2 p^{\prime}(t) u^{(n-3)}(t) u^{(n-2)}(t)-2 u^{(n-2)}(t) u^{(n-1)}(t) \\
= & 2 u^{(n-3)}(t)\left[-p(t) u^{(n-1)}(t)-q(t) u^{(n-2)}(t)-H(t, u(t)+S(t))\right] \\
& +2 p(t) u^{(n-3)}(t) u^{(n-1)}(t)+2 p(t)\left[u^{(n-2)}\right]^{2}+2 p^{\prime}(t) u^{(n-3)}(t) u^{(n-2)}(t)
\end{aligned}
$$




$$
\begin{aligned}
= & 2 u^{(n-3)}(t) u^{(n-2)}(t)\left[p^{\prime}(t)-q(t)\right]+2 p(t)\left[u^{(n-2)}(t)\right]^{2} \\
& -2 u^{(n-3)}(t) H(t, u(t)+S(t))<0 \quad \text { for } t \geq t_{6},
\end{aligned}
$$

because $0 \leq 2 q(t) \leq p^{\prime}(t)$ implies $q(t) \leq p^{\prime}(t)$. Hence $G(u(t))<0$ for $t>t_{6}$.

Now we distinguish three cases.

(i) Suppose $u^{(n-1)}(t) \geq 0$ eventually. This together with $u^{(n-2)}(t)>0$ contradicts the boundedness of $u(t)$.

(ii) Suppose $u^{(n-1)}(t) \leq 0$ for $t \geq t_{7}>t_{6}$. Since $G$ is nonincreasing, this gives us

$$
-\left[u^{(n-2)}(t)\right]^{2} \leq G(u(t)) \leq G\left(u\left(t_{7}\right)\right)<0, \quad t \geq t_{7} .
$$

So, in view of this and the fact that $u^{(n-2)}(t)$ is nonincreasing and positive, there exists a number $m>0$ such that $\lim _{t \rightarrow \infty} u^{(n-2)}(t)=m>0$. This implies that $u^{(n-3)}(t) \rightarrow \infty$ as $t \rightarrow \infty$, which is a contradiction.

(iii) Suppose that $u^{(n-1)}(t)$ changes sign for arbitrarily large $t$. Recall that $u^{(n-2)}(t)>0$ for $t \geq t_{6}$. Thus $\liminf _{t \rightarrow \infty} u^{(n-2)}(t) \geq 0$. If this limit is greater than zero, then $u^{(n-2)}(t) \geq r$ for some $r>0$. This contradicts the fact that $u^{(n-3)}(t)$ is negative. Hence

$$
\liminf _{t \rightarrow \infty} u^{(n-2)}(t)=0 .
$$

Since $u^{(n-1)}(t)$ oscillates, $u^{(n-2)}(t)$ has local extrema. Thus, there exists a sequence of local minima $a_{n}$ such that $\lim _{n \rightarrow \infty} a_{n}=\infty, \lim _{n \rightarrow \infty} u^{(n-2)}\left(a_{n}\right)=$ 0 and $u^{(n-1)}\left(a_{n}\right)=0$. Consequently, if $a_{m} \geq t_{8}>t_{6}$, we obtain

$$
-\left[u^{(n-2)}\left(a_{m}\right)\right]^{2} \leq G\left(u\left(a_{m}\right)\right) \leq G\left(u\left(t_{8}\right)\right)<0,
$$

contrary to $\lim _{n \rightarrow \infty} u^{(n-2)}\left(a_{n}\right)=0$.

Hence, since $G(u(t)) \leq 0$ prevents $u^{(n-1)}(t)$ from existing, we conclude that $G(u(t))>0$ for $t \geq t_{9} \geq t_{5}$. Also, since $u^{(n-3)}(t)<0$, we can drop the the last term in (4) and obtain

$$
u^{(n-1)}(t)+p(t) u^{(n-2)}(t)<0 \quad \text { for } t \geq t_{9} .
$$

Next, we multiply equation (2) by $t^{2}$ and integrate (the first two terms by parts) from $t_{9}$ to $t, t \geq t_{9}$, to obtain

$$
\text { (6) } \begin{aligned}
t^{2} u^{(n-1)}(t)-\left(t_{9}\right)^{2} u^{(n-1)}\left(t_{9}\right) & -2 \int_{t_{9}}^{t} s u^{(n-1)}(s) d s \\
& +p(t) t^{2} u^{(n-2)}(t)-p\left(t_{9}\right)\left(t_{9}\right)^{2} u^{(n-2)}\left(t_{9}\right) \\
& +\int_{t_{9}}^{t}\left[s^{2} q(s)-\left(s^{2} p(s)\right)^{\prime}\right] u^{(n-2)}(s) d s \\
= & -\int_{t_{9}}^{t} s^{2} H(s, u(s)+S(s)) d s
\end{aligned}
$$


Since condition (3) implies that $t^{2} q(t)-\left(t^{2} p(t)\right)^{\prime} \leq 0$, in view of (5) we can rewrite (6) as

$M-2 \int_{t_{9}}^{t} s u^{(n-1)}(s) d s>-\int_{t_{9}}^{t} s^{2} H(s, u(s)+S(s)) d s>-\int_{t_{9}}^{t} s^{2} H(s, k) d s$,

with $M$ constant. From the hypotheses, since the right hand side tends to $\infty$, so must the left hand side. Therefore

$$
\int_{t_{9}}^{\infty} t u^{(n-1)}(t) d t=-\infty
$$

Now, we rewrite (6) again, but this time we drop the fourth and sixth terms to obtain

$$
t^{2} u^{(n-1)}(t)-2 \int_{t_{9}}^{t} s u^{(n-1)}(s) d s+N>-\int_{t_{9}}^{t} s^{2} H(s, k) d s .
$$

Since the right hand side tends to $\infty$, we can write

$$
\lim _{t \rightarrow \infty}\left[t^{2} u^{(n-1)}(t)-2 \int_{t_{9}}^{t} s u^{(n-1)}(s) d s\right]=\infty .
$$

Next, we define

$$
z(t)=\int_{t_{9}}^{t} s u^{(n-1)}(s) d s .
$$

Then $z^{\prime}(t)=t u^{(n-1)}(t)$ and $\lim _{t \rightarrow \infty}\left[t z^{\prime}(t)-2 z(t)\right]=\infty$. By Lemma 1 of [8], we know that $z(t)$ must tend to either $\infty$ or $-\infty$. Since we can write

$$
\lim _{t \rightarrow \infty} z(t)=\lim _{t \rightarrow \infty} \int_{t_{9}}^{t} s u^{(n-1)}(s) d s=\lim _{t \rightarrow \infty}\left[t u^{(n-2)}(t)-u^{(n-3)}(t)\right],
$$

where the last term is positive, we must have $\lim _{t \rightarrow \infty} z(t)=\infty$. This contradicts (7). Therefore, we have a contradiction in this case as well. Hence, the proof of the theorem is complete.

THEOREM 2. Consider the differential equation $(*)$ with the following additional conditions:

(i) $n \geq 3$ is an odd integer,

(ii) $p(t) \leq 0$ and $q(t) \geq 0$ eventually, and

(iii) for any positive real constant $k$,

$$
-\int^{\infty} H(t, \pm k) d t= \pm \infty
$$

Then every bounded solution of (*) must oscillate or tend to zero. 
Note that, as in Theorem 1 , here also if $Q(t) \equiv 0$, then every bounded solution of $(*)$ must oscillate.

Proof of Theorem 2. We also argue by contradiction. Without loss of generality, we will assume that $x$ is a bounded, positive solution of $(*)$ which does not tend to zero, and we will assume that all the conditions on functions $p$ and $q$ are satisfied for $t \geq t_{0} \geq 0$. Let $u(t)=x(t)-S(t), t \geq t_{0}$. Then the Lemma above guarantees that $u^{(n-2)}(t) \leq 0$ or $u^{(n-2)}(t)>0$ eventually. In order to prove the theorem, we need to consider both cases and find a contradiction.

Case 1. We assume that $u^{(n-2)}(t) \leq 0$ for all large $t$. To obtain a contradiction we follow case 1 in the proof of Theorem 1 above.

Case 2. We assume that $u^{(n-2)}(t)>0$ for $t \geq t_{1} \geq t_{0}$. As in the proof of case 2 in Theorem 1 , we know that $u(t)>0, u^{\prime}(t)>0, u^{(n-3)}(t)<0$ and $u(t)+S(t) \geq k$ for $k>0$ constant, whenever $t \geq t_{2} \geq t_{1}$. So, now we integrate equation (2) from $t_{2}$ to $t, t \geq t_{2}$, to get

$$
\begin{aligned}
u^{(n-1)}(t)+ & p(t) u^{(n-2)}(t) \\
= & u^{(n-1)}\left(t_{2}\right)+p\left(t_{2}\right) u^{(n-2)}\left(t_{2}\right) \\
& \quad+\int_{t_{2}}^{t}\left[p^{\prime}(s)-q(s)\right] u^{(n-2)}(s) d s-\int_{t_{2}}^{t} H(s, u(s)+S(s)) d s \\
= & M+f(t)-\int_{t_{2}}^{t} H(s, u(s)+S(s)) d s,
\end{aligned}
$$

where $M$ is a constant and $f(t)$ is the first integral above. Since $f(t) \geq 0$ (note $0 \leq 2 q(t) \leq p^{\prime}(t)$ implies $q(t) \leq p^{\prime}(t)$ ), we can rewrite the above as

$$
u^{(n-1)}(t)+p(t) u^{(n-2)}(t)>M+f(t)-\int_{t_{2}}^{t} H(s, k) d s .
$$

Since $p(t) \leq 0, u^{(n-2)}(t) \geq 0$ and the right hand side tends to $\infty$, we conclude that $u^{(n-1)}(t)$ must also tend to $\infty$. Therefore, $\lim _{t \rightarrow \infty} u(t)=\infty$ implies $\lim _{t \rightarrow \infty} x(t)=\infty$, which means that $x$ is unbounded. Contradiction. Hence, the result follows.

\section{References}

[1] L. Erbe, Oscillation, nonoscillation and asymptotic behaviour for third order nonlinear differential equations, Ann. Mat. Pura Appl. 110 (1976), 373-391.

[2] J. W. Heidel, Qualitative behaviour of solutions of a third order nonlinear differential equation, Pacific J. Math. 27 (1968), 507-526. 
[3] A. G. Kartsatos, The oscillation of a forced equation implies the oscillation of the unforced equation - small forcings, J. Math. Anal. Appl. 76 (1980), 98-106.

[4] A. G. Kartsatos and W. A. Kosmala, The behaviour of an nth-order equation with two middle terms, ibid. 88 (1982), 642-664.

[5] W. A. Kosmala, Properties of solutions of the higher order differential equations, Differential Equations Appl. 2 (1989), 29-34.

[6] -, Behavior of bounded positive solutions of higher order differential equations, Hiroshima Math. J., to appear.

[7] W. A. Kosmala and W. C. Bauldry, On positive solutions of equations with two middle terms, Ann. Polon. Math. 50 (1990), 241-250.

[8] V. A. Staikos and Y. G. Sficas, Forced oscillations for differential equations of arbitrary order, J. Differential Equations 17 (1975), 1-11.

DEPARTMENT OF MATHEMATICAL SCIENCES

APPALACHIAN STATE UNIVERSITY

BOONE, NORTH CAROLINA 28608

U.S.A.

Reçu par la Rédaction le 20.4.1993

Révisé le 3.11.1993 